\title{
Moving beyond Skin-Deep
}

\section{Prabir K Chakraborty}

Department of Pathology and Department of Obstetrics and Gynaecology, The University of Oklahoma Health Sciences Center, Oklahoma City, Oklahoma, USA

Corresponding Author: Chakraborty PK, Department of Pathology and Department of Obstetrics and Gynaecology, The University of Oklahoma Health Sciences Center, Oklahoma City, Oklahoma, USA, Tel: 405 271 7184; Email Id: Prabir-Chakraborty@ouhsc.edu

Received date: November 03, 2015; Accepted date: December 19, 2015; Published date: January 04, 2016

Copyright: (C) 2016 Chakraborty PK. This is an open-access article distributed under the terms of the Creative Commons Attribution License, which permits unrestricted use, distribution, and reproduction in any medium, provided the original author and source are credited.

\section{Moving beyond Skin-Deep}

The skin is an essential organ with diverse functions. A deep molecular understanding to the biology of skin can offer explanations to many dermatological diseases. Furthermore, the skin perhaps serves the best model for cell biology research ranging from adult stem cell research to general questions in cancer and in development. The skin offers a multiple advantages to be used as a model system that other tissues do not possess. Advantages of skin as a study model include: simple visualization, large size, less complex cell culture systems, and efficient physiological readouts[1]. Here, I would address two recent findings in the field of stem cell biology using the skin as model system, and this might bring forward revolutionary outcomes in the biomedical field.

A seminal article from Dr. Buganim (IMRIC's Department of Developmental Biology and Cancer Research) titled "Extensive Nuclear Reprogramming Underlies Lineage Conversion into Functional Trophoblast Stem-like Cells' successfully converted skin cells into durable and fully functional placenta-generating cells more appropriately termed as induced trophoblast stem cells ("iTSCs")[2].

The placenta is crucial for the health of the mother and her fetus. Unfortunately our understanding is still elusive for the placenta. Improper development or damage to the placenta is correlated with recurrent miscarriage, low birth weight, premature birth and birth defects. Unfortunately present options to model or treat these disorders are sparse since in vitro isolation and propagation of placental cells have failed. Earlier in 2006, Nobel laureate Shinya Yamanaka and Kazutoshi Takahashi, have deciphered four genes that can be introduced in skin cells to reprogram fibroblasts into functional embryonic stem-like cells known as "induced pluripotent stem cells" ("iPSCs")[3]. On this line, Dr. Buganim and colleagues attempted to generate stable and functional placenta-generating cells. In the mouse model they screened for genes that support the development of the placenta and identified three genes (Gata3, Eomes and Tfap2c) that could reprogram a cell fate that completely converted the skin cells into stable and fully functional placenta-generating cells. These skinderived TSCs had similar morphology to wild type TSCs, and could contribute to developing placenta[2]. Moreover these cells do not risk the embryo since they integrate only into the placenta. The success of this study paves a great opportunity to develop actual chance for women who suffer from placental dysfunction diseases to have healthy babies[4].
But this gene manipulation approach has drawbacks. It involves complex and time-consuming, steps and there lies a chance of the inserted gene to integrate non-specifically in the chromosome and generate lethal mutations or cancer activating genes. In this context, a team of researchers from The Chinese Academy of Sciences in Shanghai (China) have found a unique way to bring about the same biological metamorphosis: turning skin cells into neurons. The approach involved simply adding a few chemicals to skin cells. The secret lies in the ability of these chemicals to change the activity of particular genes that execute the transformation[5].

They probed thousands of chemicals and identified a specific composition of molecules that essentially switched off skin cell genes in human cells and turned on neuron genes. Specifically the recipe consisted of seven small molecules referred as VCRFSGY (valproic acid, CHIR99021, Repsox, Forskolin, SP600125, GO6983, and Y-27632). When these chemicals were put together in culture dishes of human skin cells, within few weeks the cells transformed into mature and functional neurons. Intriguingly, they showed that skin cells from an Alzheimer's patient could be molded into neurons that expressed several critical markers of Alzheimer's disease[5]. These results will immensely contribute to the future of Alzheimer's research by providing scientists a safe and accurate platform to test the effects of possible new drugs

In summary, these examples establish that the skin serves as a powerful tool in which one can examine a wide array of cell biological phenomena and holds the holy grail of stem cell research for novel therapeutic outcomes.

\section{References}

1. Morrow A, Lechler T (2015) Studying cell biology in the skin. Mol biol cell 26: 4183-4186.

2. Benchetrit H, Herman S, Van Wietmarschen N, Wu T, Makedonski K, et al. (2015) Extensive Nuclear Reprogramming Underlies Lineage Conversion into Functional Trophoblast Stem-like Cells, Cell stem cell 17: 543-556.

3. Takahashi K, Yamanaka S (2006) Induction of pluripotent stem cells from mouse embryonic and adult fibroblast cultures by defined factors, Cell 126: 663-676.

4. http://new.huji.ac.il/en/article/27928.

5. Hu W, Qiu B, Guan W, Wang Q, Wang M, et al. (2015) Direct Conversion of Normal and Alzheimer's Disease Human Fibroblasts into Neuronal Cells by Small Molecules, Cell stem cell 17: 204-212. 